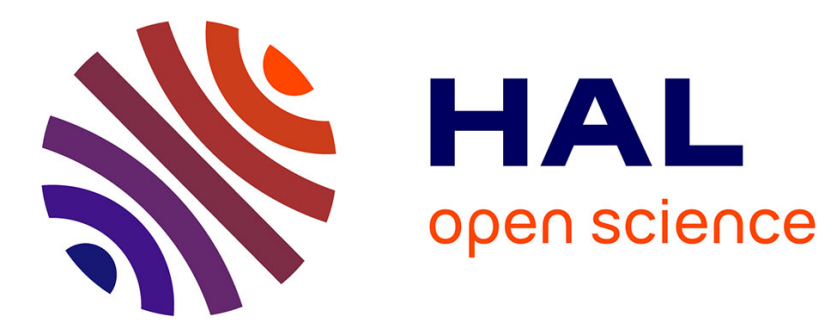

\title{
Dynamical Slowdown of Polymers in Laminar and Random Flows
}

\author{
Antonio Celani, Alberto Puliafito, Dario Vincenzi
}

\section{To cite this version:}

Antonio Celani, Alberto Puliafito, Dario Vincenzi. Dynamical Slowdown of Polymers in Laminar and Random Flows. Physical Review Letters, 2006, 97, pp.118301. 10.1103/PhysRevLett.97.118301. hal-00097741

\section{HAL Id: hal-00097741 \\ https://hal.science/hal-00097741}

Submitted on 22 Sep 2006

HAL is a multi-disciplinary open access archive for the deposit and dissemination of scientific research documents, whether they are published or not. The documents may come from teaching and research institutions in France or abroad, or from public or private research centers.
L'archive ouverte pluridisciplinaire HAL, est destinée au dépôt et à la diffusion de documents scientifiques de niveau recherche, publiés ou non, émanant des établissements d'enseignement et de recherche français ou étrangers, des laboratoires publics ou privés. 


\title{
Dynamical slowdown of polymers in laminar and random flows
}

\author{
A. Celani, ${ }^{1}$ A. Puliafito, ${ }^{1}$ and D. Vincenzi ${ }^{2,3}$ \\ ${ }^{1}$ CNRS-INLN, 1361 Route des Lucioles, 06560 Valbonne, France \\ ${ }^{2}$ Max-Planck-Institut für Dynamik und Selbstorganisation, Bunsenstraße 10, 37073 Göttingen, Germany \\ ${ }^{3}$ Sibley School of Mechanical 83 Aerospace Engineering and LASSP, Cornell University, Ithaca, NY 14853
}

\begin{abstract}
The influence of an external flow on the relaxation dynamics of a single polymer is investigated theoretically and numerically. We show that a pronounced dynamical slowdown occurs in the vicinity of the coil-stretch transition, especially when the dependence on polymer conformation of the drag is accounted for. For the elongational flow, relaxation times are exceedingly larger than the Zimm relaxation time, resulting in the observation of conformation hysteresis. For random smooth flows hysteresis is not present. Yet, relaxation dynamics is significantly slowed down because of the large variety of accessible polymer configurations. The implications of these results for the modeling of dilute polymer solutions in turbulent flows are addressed.
\end{abstract}

Phys. Rev. Lett. 97, 118301 (2006)

http://link.aps.org/abstract/PRL/v97/e118301

The dynamics of an isolated polymer in a flow field forms the basis of constitutive models for dilute polymer solutions [1, 2, 3]. The modeling of drag-reducing flows, for instance, requires an appropriate description of single polymer deformation in turbulent velocity fields [ 4 . In the last decade, major advances in fluorescence microscopy offered the possibility of tracking isolated polymers both in laminar [3] and random flows [5]. The dynamics of a polymer in thermal equilibrium with the surrounding solvent is commonly described in terms of normal modes and relaxation times associated with them. The analytical form of the relaxation spectrum was first obtained by Rouse under the assumption that the polymer could be described as a series of beads connected by Hookean springs [6]. The Rouse model was subsequently improved by Zimm to include hydrodynamical interactions between segments of the polymer [7]. In Zimm's formulation, the equations of motion are decoupled into a normal mode structure by preaveraging the distances between the beads over the distribution of polymer configurations. The relaxation time associated with the fundamental mode, $\tau$, determines the typical time that it takes for a deformed polymer to recover the equilibrium configuration in a solvent. The normal mode theory was confirmed by the analysis of the oscillatory motion of a DNA molecule immersed in a solvent and held in a partially extended state by means of optical tweezers 80. An alternative approach to examine polymer relaxation consists in stretching a tethered DNA molecule in a flow and measuring its relaxation after cessation of the flow [9, 10. The theoretical predictions for these experimental conditions are provided by the scaling theory [11 and the static dynamics formalism 12].

The aforementioned studies all consider the internal dynamics of a polymer floating in a solvent under the influence of thermal noise only - the interaction of the polymer with an external flow is not taken into account. One of the aspects highlighted by experiments is that polymer dynamics in a moving fluid is strongly influenced by the carrier velocity field. The coil-stretch transition is the most noticeable example: as the strain rate exceeds a threshold value, the polymer undergoes a transition from the coiled, equilibrium configuration to an almost fully extended one [13]. Therefore, when a polymer is freely transported by a non-uniform flow, we expect that the time scales describing its dynamics may be significantly different from the Zimm time $\tau$. Simple models for polymer stretching indeed suggest deviations from Zimm's theory near the coil-stretch transition [14, 15, 16]. Discrepancies in the definition of the correct relaxation time are also encountered in drop formation experiments [17].

In this Letter we investigate polymer relaxation dynamics both in elongational and random smooth flows. Our analysis brings to evidence an important slowdown of dynamics with respect to the Zimm timescales, in the vicinity of the coil-stretch transition. For the elongational flow, this is related to conformation hysteresis 18, 19, 20. For random flows, we show that hysteresis is not present. Nonetheless, the amplification of the relaxation time persists, albeit to a lesser extent, due to the large heterogeneity of polymer configurations. In both cases, the dependence of the drag force on the polymer configuration plays a prominent role. This suggests the necessity of improving current models of polymer solutions in turbulent flows to account for such effect.

The dumbbell approximation is the basis of the most common models of single polymer dynamics and viscoelastic models of dilute polymer solutions [21]. Its validity relies on the fact that the slowest deformational mode of the polymer is the most influential in producing viscoelasticity [4]. However, when attention is directed to non-equilibrium dynamics it is often too crude to assume that $\tau$ is independent of the conformation of the molecule [22. Therefore, following de Gennes [13], Hinch [23], and Tanner's 24] approach, we consider a model where the polymer is described as two beads con- 
nected by an elastic spring and the probability density function of the end-to-end vector, $\Psi(\boldsymbol{R}, t)$, satisfies the diffusion equation:

$$
\frac{\partial \Psi}{\partial t}=-\frac{\partial}{\partial \boldsymbol{R}} \cdot\left[\boldsymbol{\kappa}(t) \cdot \boldsymbol{R} \Psi-\frac{f(R) \boldsymbol{R}}{2 \tau \nu(R)} \Psi-\frac{R_{0}^{2}}{2 \tau \nu(R)} \frac{\partial \Psi}{\partial \boldsymbol{R}}\right]
$$

where $\kappa_{i j}(t)=\partial_{j} v_{i}(t)$ is the velocity gradient, $R_{0}$ is the mean extension at thermal equilibrium and $R=|\boldsymbol{R}|$. The function $f(R)$ defines the entropic force restoring stretched molecules into the coiled configuration. Synthetic polymers are properly described by the Warner law, $f(R)=1 /\left(1-R^{2} / L^{2}\right)$, where $L$ is the contour length of the polymer; biological macromolecules are better characterized by the Marko-Siggia law, $f(R)=$ $2 / 3-L /(6 R)+L /\left[6 R(1-R / L)^{2}\right]$ [2]. The flow strength relative to the polymer tendency to recoil is expressed by the Weissenberg number $W i$, defined as the product of the Zimm time $\tau$ by a characteristic extension rate of the flow. The function $\nu(R)$ encodes for the dependence on the polymer conformation of the drag exerted by the fluid: a spherical coil offers a smaller resistance with respect to a long rod-like configuration. We utilize the expression $\nu(R)=1+\left(\zeta_{s} / \zeta_{c}-1\right) R / L$ that interpolates linearly between these extremes (see Refs. [25, 26]). Here, $\zeta_{c}=3 \sqrt{6 \pi^{3}} R_{0} \eta / 8$ and $\zeta_{s}=2 \pi L \eta / \ln (L / \ell)$ are the friction coefficients for the coiled and the stretched configuration, respectively, $\eta$ is the solvent viscosity and $\ell$ is the diameter of the molecule. Strictly speaking, the above form of $\nu(R)$ was deduced from experiments and microscopic simulations of laminar flows. To our knowledge, measures of $\nu(R)$ in random flows are not available yet, and the study of its functional dependence lies beyond the scope of the present work. Assuming that the polymer is aligned with the local stretching direction of the flow for the most part of its evolution, we shall use a linear $\nu(R)$ for a random flow as well.

To define the relaxation time in the presence of an arbitrary external flow, we consider the probability density function of the rescaled extension, $P(r, t)$ with $r=R / L$, and identify the dynamical relaxation time, $t_{\text {rel }}$, as the characteristic time needed for $P(r, t)$ to attain its stationary form $P_{\mathrm{st}}(r)$. In the cases examined in this Letter, as we shall see, the probability density function of $r$ satisfies the Fokker-Planck equation

$$
\partial_{t^{\prime}} P=-\partial_{r}\left(D_{1}(r) P\right)+\partial_{r} D_{2}(r) \partial_{r} P,
$$

where the form of $D_{1}(r)$ and $D_{2}(r)$ depends on the flow and $t^{\prime}=t / \tau$. The stationary solution to Eq. (2) takes the potential form $P_{\mathrm{st}}(r)=N \exp \left[-E(r) / K_{B} T\right]$, where $N$ is the normalization constant and $E(r)=$ $-K_{B} T \int^{r} D_{1}(\rho) / D_{2}(\rho) d \rho$. The finite-time solution admits the expansion

$$
P\left(r, t^{\prime}\right)=P_{\mathrm{st}}(r)+\sum_{n=1}^{\infty} a_{n} p_{n}(r) e^{-t^{\prime} / \sigma_{n}}
$$
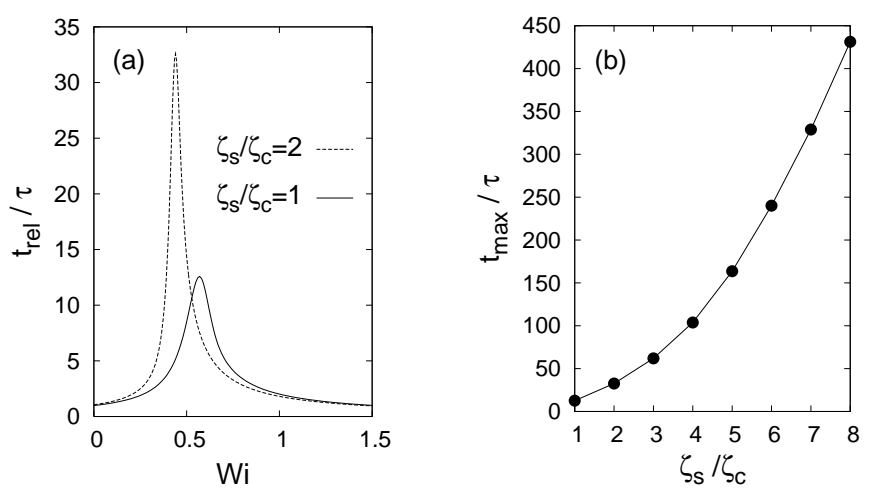

Figure 1: Elongational flow: (a) rescaled relaxation time vs. $W i$ for $b=400$. The entropic force is given by the Warner law. For small $W i, t_{\text {rel }} / \tau$ grows as $1 /(1-2 W i)$, as can be seen by replacing $\hat{f}(r)$ with 1 in Eq. (2). For $W i \gg W i_{\text {crit }}$, the time needed to reach the asymptotic regime is set by the time scale of the flow, $\gamma^{-1}$, and $t_{\mathrm{rel}} / \tau$ decreases as $W i^{-1}$. (b) Rescaled maximum relaxation time $t_{\max } / \tau$ vs. $\zeta_{s} / \zeta_{c}(b=400)$.

where the coefficients $a_{n}$ are fixed by $P(r, 0), p_{n}(r)$ are the eigenfunctions of the Fokker-Planck operator, and $\sigma_{n}$ are the reciprocals of its (strictly positive) eigenvalues, arranged in descending order $\left(\sigma_{n}>\sigma_{n+1}\right)$. The relaxation time is thus defined as $t_{\mathrm{rel}} \equiv \sigma_{1} \tau$.

As a first example, we examine the steady planar elongational flow $\boldsymbol{v}=\gamma(x,-y)$. By assuming that the polymer extension in the $x$-direction is much greater than in the $y$-direction, it is easy to derive from Eq. (1) an equation of the form (2) with $D_{1}(r)=W i r-\hat{f}(r) r /[2 \hat{\nu}(r)]$, $D_{2}(r)=[2 b \hat{\nu}(r)]^{-1}, \hat{f}(r)=f(r L), \hat{\nu}(r)=\nu(r L)$, $b=L^{2} / R_{0}^{2}$, and $W i=\gamma \tau$ [27]. For $\zeta_{s}=\zeta_{c}, t_{\text {rel }}$ can be computed from (33) by solving a central two-point connection problem for a generalized spheroidal wave equation [16]. In the general case, $\zeta_{s}>\zeta_{c}$, we resorted to a numerical computation based on the variation-iteration method of quantum mechanics [28]. In the vicinity of the coil-stretch transition $(W i=1 / 2) t_{\text {rel }}$ shows a sharp peak (Fig. 1). In this range of Wi there is a critical competition between the entropic force and the velocity gradient that makes the convergence time to the steady state extremely long. This effect is strongly enhanced by the conformation-dependent drag; the peak $t_{\max }$ indeed increases with $\zeta_{s} / \zeta_{c}$ (Fig. I). Those extremely long relaxation times are intimately connected with the observation of finite-time conformation hysteresis 118, 19, 20. For large enough $\zeta_{s} / \zeta_{c}$, there is a narrow range of $W i$ around the critical value for the coil-stretch transition where $E(r)$ has a double well structure [18, 19, 20]. The barrier height separating the coiled and the stretched state is much greater than the thermal energy $K_{B} T$, and therefore the polymer remains trapped in its initial configuration for an exceptionally long time (Fig. 2).

The discovery of elastic turbulence has recently allowed the examination of single polymer dynamics in a 

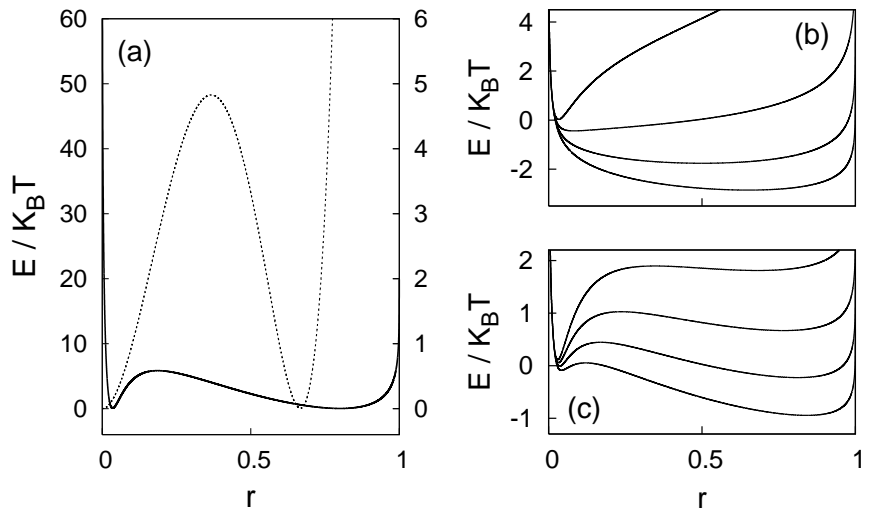

Figure 2: (a) Effective energy at the coil-stretch transition for a polyacrylamide (PAM) molecule $\left(b=3953, \zeta_{s} / \zeta_{c}=6.87\right)$ in the elongational flow (dashed line) and the 3D BatchelorKraichnan flow (solid line). The left vertical axis refers to the dashed line; the right vertical axis refers to the solid line; (b) effective energy in the random flow for a PAM molecule with constant drag $\left(\zeta_{s}=\zeta_{c}\right)$ (from top to bottom $W i=$ $0.4,0.7,1.0,1.3)$; (c) the same as (b), but with $\zeta_{s}=6.87 \zeta_{c}$ (from top to bottom $W i=0.28,0.33,0.38,0.43$ ). When $\zeta_{s}>$ $\zeta_{c}$ the transition occurs in a much narrower range of $W i$.

random smooth flow generated by viscoelastic instabilities [5, 29]. The velocity gradient fluctuates along fluid trajectories; the average local deformation rates define the three Lyapunov exponents of the flow. Experimental observations have been accompanied by theoretical and numerical studies based on the dumbbell model (see, e.g., Refs. [14, 15, 30]). To analytically investigate polymer relaxation dynamics in random flows, we initially assume that the velocity field obeys the BatchelorKraichnan statistics [31]. The velocity gradient is then a statistically isotropic and parity invariant Gaussian process with zero mean and correlation: $\left\langle\kappa_{i j}(t) \kappa_{k l}(s)\right\rangle=$ $2 \lambda \delta(t-s)\left[(d+1) \delta_{i k} \delta_{j l}-\delta_{i j} \delta_{k l}-\delta_{i l} \delta_{j k}\right] /[d(d-1)]$, where $d$ is the dimension of the flow and $\lambda$ denotes the largest Lyapunov exponent. In this context, we indicate by $P\left(r, t^{\prime}\right)$ the probability density function of the extension both with respect to thermal noise and the realizations of the velocity field. For the elongational flow Eq. (2) was obtained under the uniaxial approximation. On the contrary, for the isotropic random flow Eq. (2) holds exactly and can be obtained by a Gaussian integration by parts followed by integration over angular variables. The drift and diffusion coefficients take the form $D_{1}(r)=$ $(d-1) / d W i r-\hat{f}(r) r /[2 \hat{\nu}(r)]+(d-1) /[2 b \hat{\nu}(r) r]$ and $D_{2}(r)=W i r^{2} / d+[2 b \hat{\nu}(r)]^{-1}$ with $W i=\lambda \tau$. The stationary distribution admits once more a potential form. For $d=3$ and $\zeta_{s}>\zeta_{c}$, the potential displays a very wide well, the effect of the conformation-dependent drag being to increase the probability of large extensions. There is no evidence of pronounced double wells (Fig. 2). Near the coil-stretch transition, the effective barrier heights separating the coiled and stretched states are indeed at most comparable to thermal energy. For realistic $\zeta_{s} / \zeta_{c}$ no conformation hysteresis is therefore expected to be observed in random flows. The behavior of $t_{\text {rel }}$ vs. $W i$ is however analogous to the one encountered in the elongational flow: $t_{\text {rel }} / \tau$ increases as $[1-W i(d+2) / d]^{-1}$ at small $W i$ and decreases as $W i^{-1}$ at large $W i$. A peak near the transition is present, that becomes more and more pronounced with increasing $\zeta_{s} / \zeta_{c}$, attaining values as large as about thirty times $\tau$ (Fig. 3). The reason for this behavior is the breadth of $P_{\mathrm{st}}(r)$ and the consequent large heterogeneity of accessible polymer configurations.

To corroborate the results obtained in the context of the short-correlated flow, we performed Brownian Dynamics simulations of dumbbell molecules [32] in the random flow introduced by Brunk et al. 34. This model reproduces the small-scale structure of a turbulent flow by means of a statistically isotropic Gaussian velocity gradient. The autocorrelation times of components of the strain and rotation tensors are set to be multiple of the Kolmogorov time $\tau_{\eta}$ by comparison with direct numerical simulations of $3 \mathrm{D}$ isotropic turbulence $\left(\tau_{S}=2.3 \tau_{\eta}\right.$, $\left.\tau_{R}=7.2 \tau_{\eta}\right)$. The Lyapunov exponent of this flow is $\lambda \simeq 10 \tau_{\eta}^{-1}$. We computed $t_{\text {rel }}$ as the time of convergence of the moments $\left\langle r^{n}(t)\right\rangle$ to their stationary value $\left\langle r^{n}\right\rangle_{\mathrm{st}}$ : $t_{\text {rel }}^{-1}=-\lim _{t \rightarrow \infty} \ln \left[\left\langle r^{n}(t)\right\rangle-\left\langle r^{n}\right\rangle_{\mathrm{st}}\right] / t$, where the averages were taken over an ensemble of realizations of the flow, all with the same initial extension $r(0)$. The numerical difficulty arising from the singularity of the entropic force at $R=L$ has been overcome by exploiting the algorithm introduced in [35]. The results shown in Fig. 1 confirm the scenario depicted in the context of the
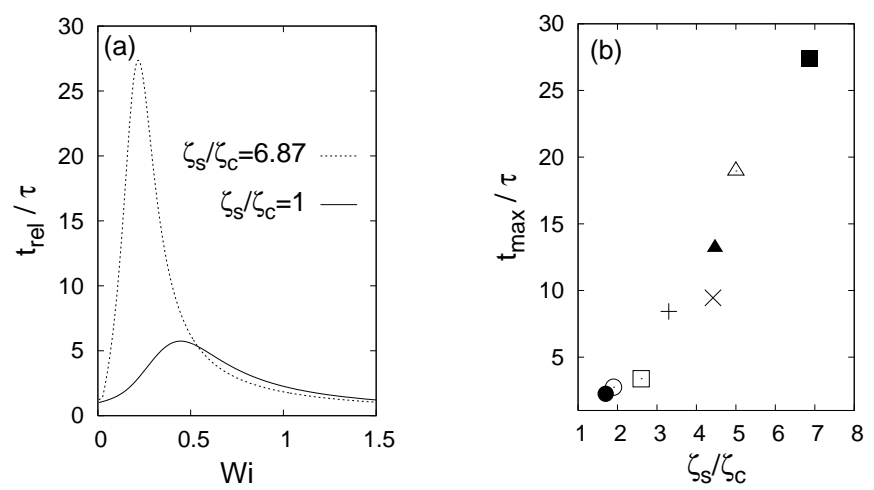

Figure 3: 3D Batchelor-Kraichnan flow: (a) $t_{\text {rel }} / \tau$ vs. $W i$ for a PAM molecule $(b=3953)$; (b) $t_{\max } / \tau$ for the following polymers: DNA $(\bullet, b=191.5 ; \circ, b=260 ; \square, b=565 ;+, b=$ $2250)$, polystyrene $(\times, b=673)$, polyethyleneoxide (PEO) $(\Lambda, b=1666)$, Escherichia Coli DNA $(\triangle, b=9250)$, PAM (a). Measures of $b$ and $\zeta_{s} / \zeta_{c}$ can be found in [18, 19, 20, 25]. Synthetic polymers are modeled by the Warner law, whereas biological molecules are described by the Marko-Siggia law. Relaxation times were computed by means of the variationiteration method [28]. For $\zeta_{s}=\zeta_{c}$ they can be obtained by solving an eigenvalue problem for a Heun equation 15 . 

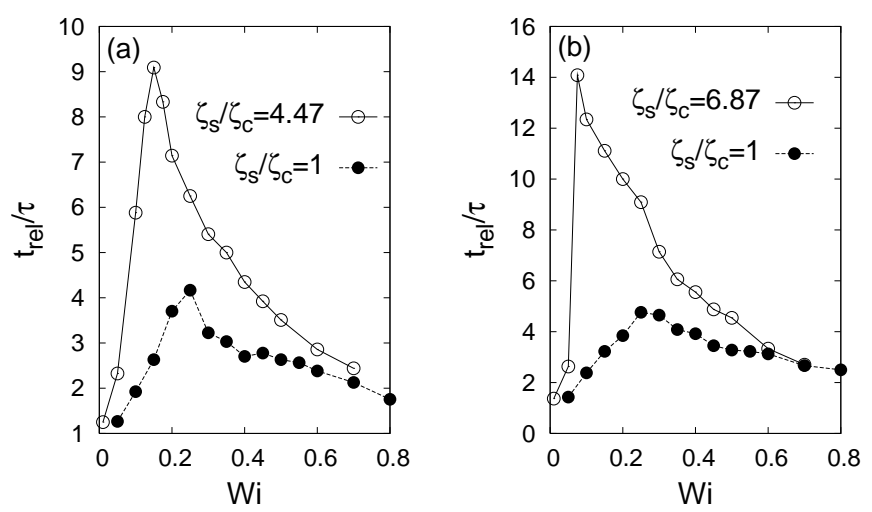

Figure 4: Brunk-Koch-Lion flow: $t_{\text {rel }} / \tau$ vs. $W i=\lambda \tau$ from Brownian Dynamics simulations; (a) PEO; (b) PAM.

short-correlated flow. It is worth noting that the above definition provides an operational method to measure $t_{\text {rel }}$ that can be implemented in experiments.

In summary, we have shown that the equilibrium configuration of a polymer in a flow, as well as the time a deformed molecule takes on average to recover that configuration, depend sensitively on the properties of the flow. In the vicinity of the coil-stretch transition the characteristic relaxation time is much longer than the Zimm time, both in elongational and random flows. In other words, the effective $W i$ differs considerably from the "bare" one 36]. This effect is strongly amplified when the drag coefficient depends on the conformation of the polymer, and may play an important role in drag-reducing turbulent flows, where the strain rate often fluctuates around values typical of the coil-stretch transition 38. Our conclusions thus suggest that the conformation-dependent drag should be included as a basic ingredient of continuum models of polymer solutions, calling for further theoretical, experimental and numerical study.

The authors gratefully acknowledge inspiring discussions with M. Chertkov, S. Gerashchenko, M. Martins Afonso and V. Steinberg. This work has been partially supported by the EU (contract HPRN-CT-2002-00300).

[1] S. Chu, Phil. Trans. R. Soc. Lond. A 361, 689 (2003).

[2] R. G. Larson, J. Rheol. 49, 1 (2005).

[3] E. S. G. Shaqfeh, J. Non-Newton. Fluid Mech. 130, 1 (2005)

[4] A. Gyr and H. W. Bewersdorff, Drag reduction of Turbulent Flows by Additives (Kluwer Academic, Dordrecht, Boston, 1995)

[5] S. Gerashchenko, C. Chevallard, and V. Steinberg, Europhys. Lett. 71, 221 (2005).

[6] P. E. Rouse, Jr., J. Chem. Phys. 21, 1272 (1953).

[7] B. H. Zimm, J. Chem. Phys. 24, 269 (1956).

[8] S. R. Quake, H. Babcock, and S. Chu, Nature 388, 151 (1997).
[9] T. T. Perkins et al., Science 264, 822 (1994).

[10] S. Manneville et al., Europhys. Lett. 36, 413 (1996).

[11] Y. Marciano and F. Brochard-Wyart, Macromolecules 28, 985 (1995)

[12] R. Rzehak and W. Zimmermann, Europhys. Lett. 59, 779 (2002).

[13] P. G. de Gennes, J. Chem. Phys. 60, 5030 (1974).

[14] A. Celani, S. Musacchio, and D. Vincenzi, J. Stat. Phys. 118, 531 (2005).

[15] M. Martins Afonso and D. Vincenzi, J. Fluid Mech. 540, 99 (2005).

[16] D. Vincenzi and E. Bodenschatz, J. Phys. A: Math. Gen. 39, 10691 (2006)

[17] V. Tirtaatmadja, G. H. McKinley, and J. J. CooperWhite, Phys. Fluids 18, 043101 (2006).

[18] C. M. Schroeder et al., Science 301, 1515 (2003).

[19] C. M. Schroeder, E. S. G. Shaqfeh, and S. Chu, Macromolecules 37, 9242 (2004).

[20] C.-C. Hsieh and R. G. Larson, J. Rheol. 49, 1081 (2005).

[21] R. B. Bird et al., Dynamics of Polymeric Liquids, vol. 2, 2nd edition (Wiley, New York, 1987).

[22] J. W. Hatfield and S. R. Quake, Phys. Rev. Lett. 82, 3548 (1999).

[23] E. J. Hinch, in Proceedings of Colloques Internationaux du CNRS No. 233 (CNRS Editions, Paris, 1974), p. 241.

[24] R. I. Tanner, Trans. Soc. Rheol. 19, 557 (1975)

[25] R. G. Larson et al., Phys. Rev. E 55, 1794 (1997).

[26] P. S. Doyle et al., J. Non-Newton. Fluid Mech. 76, 79 (1998).

[27] Our definition of $W i$ follows the literature on elongational flows and therefore is half the one commonly adopted in the context of random flows.

[28] P. M. Morse and H. Feshbach, Methods of Theoretical Physics (McGraw-Hill, New York, 1953).

[29] A. Groisman and V. Steinberg, Nature 405, 53 (2000); New J. Phys. 6, 29 (2004); T. Burghelea, E. Segre and V. Steinberg, Phys. Fluids 17, 103101 (2005).

[30] M. Chertkov, Phys. Rev. Lett. 84, 4761 (2000); E. Balkovsky, A. Fouxon, and V. Lebedev, Phys. Rev. Lett. 84, 4765 (2000); J.-L. Thiffeault, Phys. Lett. A 308, 445 (2003); M. Chertkov et al., J. Fluid Mech. 531, 251 (2005); A. Puliafito and K. Turitsyn, Physica D 211, 9 (2005); J. Davoudi and J. Schumacher, Phys. Fluids 18, 025103 (2006).

[31] R. H. Kraichnan, Phys. Fluids 11, 945 (1968).

[32] The integration algorithm is based on the Itô stochastic differential equation equivalent to Eq. (1),$d \boldsymbol{R}=$ $\left\{\boldsymbol{\kappa}(t) \cdot \boldsymbol{R}-f(R) \boldsymbol{R} /[2 \tau \nu(R)]-R_{0}^{2} \nu^{\prime}(R) \boldsymbol{R} /\left[2 \tau \nu^{2}(R) R\right]\right\} d t+$ $\sqrt{R_{0}^{2} /[\tau \nu(R)]} d \boldsymbol{W}(t)$ 33]; $\boldsymbol{W}(t)$ is the Brownian motion.

[33] H. C. Öttinger, Stochastic Processes in Polymeric Fluids (Springer-Verlag, Berlin, Heidelberg, 1996).

[34] B. K. Brunk, D. L. Koch, and L. W. Lion, Phys. Fluids 9, 2670 (1997); J. Fluid Mech. 364, 81 (1998).

[35] A. Celani, A. Puliafito, and K. Turitsyn, Europhys. Lett. 70, 464 (2005).

[36] This fact is related to the overestimation of the drag reducing $W i$ encountered in numerical simulations 337 .

[37] Y. Dubief et al., J. Fluid Mech. 514, 271 (2004).

[38] K. R. Sreenivasan and C. M. White, J. Fluid Mech. 409, 149 (2000); E. Balkovsky, A. Fouxon, and V. Lebedev, Phys. Rev. E 64, 056301 (2001); G. Boffetta, A. Celani, and S. Musacchio, Phys. Rev. Lett. 91, 034501 (2003); V. S. L'vov et al., ibid. 92, 244503 (2004). 